\title{
Validity and completeness of rheumatoid arthritis diagnoses in the nationwide DANBIO clinical register and the Danish National Patient Registry
}

This article was published in the following Dove Press journal:

Clinical Epidemiology

Else Helene Ibfelt,' Jan Sørensen, ${ }^{2,3}$ Dorte V Jensen ${ }^{4,5}$ Lene Dreyer 5,6 Berit Schiøttz-Christensen, ${ }^{7}$ Pia H Thygesen, ${ }^{8}$ Ada Colic, ${ }^{9}$ Johnny L Raun, ${ }^{10}$ Natalia Manilo," Anne Rødgaard, ${ }^{4}$ Uta E Poulsen, ${ }^{12}$ Claus Rasmussen, ${ }^{13}$ Torben Hansen, ${ }^{14}$ Babara Unger, ${ }^{15}$ Randi Pelck, ${ }^{16}$ Anita Kincses, ${ }^{17}$ Henrik Nordin, ${ }^{18}$ Tove Lorenzen, ${ }^{19} \mathrm{Ali}$ Theibich, ${ }^{20}$ Inger Marie Jensen Hansen, ${ }^{21}$ Jakob Espesen, ${ }^{22}$ Jolanta Grydehøj, ${ }^{23}$ Mette Holland-Fischer, ${ }^{24}$ Anne Gitte Loft, ${ }^{25}$ Merete Lund Hetland ${ }^{4,26}$

'Research Centre for Prevention and Health, Capital Region of Denmark, Rigshospitalet - Glostrup, Denmark; ${ }^{2}$ Centre of Health Economics Research, Institute of Public health, University of Southern Denmark, Odense, Denmark; ${ }^{3}$ Healthcare Outcome Research Centre, Royal College of Surgeons in Ireland, Dublin, Ireland; ${ }^{4} \mathrm{DANBiO}$ and Copenhagen Center for Arthritis Research, Center for

Rheumatology and Spine Diseases, Rigshospitalet Glostrup, Glostrup, ${ }^{5}$ Center for Rheumatology and Spine Diseases, Gentofte University Hospital, Hellerup, ${ }^{6}$ The Parker Institute, Frederiksberg and Bispebjerg Hospital, Frederiksberg, ${ }^{7}$ Spine Centre of Southern Denmark, Hospital Lillebaelt, Middelfart, ${ }^{8}$ Department of Rheumatology, Odense University Hospital, Odense, 'Department of Rheumatology, Sydvestiysk Sygehus, Esbjerg/ Rheumatology, Sydvestysk Sygehus, Esbjerg/ ( Fredericia, "Department of Rheumatology, Frederiksberg Hospital, Copenhagen, ${ }^{12}$ Department of Rheumatology, Gigthospital Gråsten, Gråsten, ${ }^{13}$ Clinic of Internal Medicine, Rheumatology, Regionshospital Nordjylland, Hjørring, ${ }^{14}$ Department of Rheumatology, Holbæk sygehus, Holbrk, ${ }^{15}$ Department of Internal Medicine/Rheumatology, Hospitalsenheden Horsens, Horsens, ${ }^{16}$ Department of Rheumatology, Zealand University Hospital, Køge, ${ }^{17}$ Department of Rheumatology, Nordsjællands Hospitaler, Hillerød, ${ }^{18}$ Center for Rheumatology and Spine Diseases, Centre of Head and Orthopedics, Rigshospitalet, Copenhagen, ${ }^{19}$ Department of Rheumatology Silkeborg Regional Hospital and University Clinic, Silkeborg, keborg, ${ }^{20}$ Department of Rheumatology, Slagelse Hospital, Slagelse, ${ }^{21}$ Department of Rheumatology and University of Southern Denmark, Svendborg Hospital, Odense, ${ }^{22}$ Department of Internal Medicine, Vejle Hospital, Vejle, ${ }^{23}$ Department of Rheumatology, Regional Hospital, West Jutland, Herning, ${ }^{24}$ Department of Rheumatology, Aalborg University Hospital, Ålbor ${ }^{25}$ Department of Rheumatology, Aarhus University Depart Hospital, Aarhus, 'Dise Faculty of Health and Medical Sciences, University of Copenhagen, Copenhagen, Denmark

Correspondence: Else Helene Ibfelt Research Center for Prevention and Health, Capital Region of Denmark, Rigshospitalet - Glostrup, Nordre Ringvej 57, 2600 Glostrup, Denmark

$\mathrm{Tel}+4529214068$

Email elsibf@rkkp.dk
Objectives: In Denmark, patients with rheumatoid arthritis (RA) are registered in the nationwide clinical DANBIO quality register and the Danish National Patient Registry (DNPR). The aim was to study the validity of the RA diagnosis and to estimate the completeness of relevant RA cases in each registry.

Study design and setting: Patients registered for the first time in 2011 with a diagnosis of RA were identified in DANBIO and DNPR in January 2013. For DNPR, filters were applied to reduce false-positive cases. The diagnosis was verified by a review of patient records. We calculated the positive predictive values (PPVs) of the RA diagnosis registrations in DANBIO and DNPR, and estimated the registry completeness of relevant RA cases for both DANBIO and DNPR. Updated data from 2011 to 2015 from DANBIO were retrieved to identify patients with delayed registration, and the registry completeness and PPV was recalculated.

Results: We identified 1,678 unique patients in DANBIO or in DNPR. The PPV (2013 dataset) was $92 \%$ in DANBIO and $79 \%$ in DNPR. PPV for DANBIO on the 2015 update was $96 \%$. The registry completeness of relevant RA cases was $43 \%$ in DANBIO, increasing to $91 \%$ in the 2015 update and $90 \%$ in DNPR.

Conclusion: DANBIO held a high proportion of true RA cases $(96 \%)$ and was found to be superior to the DNPR (79\%) with regard to the validity of the diagnosis. Both registries were estimated to have a high completeness of RA cases treated in hospital care ( $90 \%)$.

Keywords: rheumatoid arthritis, validity, incidence, clinical registry, Denmark

\section{Introduction}

Rheumatoid arthritis (RA) is a chronic autoimmune disease, typically involving the small joints of hands and feet. The disease requires lifelong monitoring and treatment. In the majority of patients, RA limits daily functioning, quality of life, and the ability to maintain work, and it imposes high welfare costs as a consequence. ${ }^{1,2}$ The prevalence of RA in Denmark has been estimated to be $0.5 \%-0.9 \%{ }^{3}$

In clinical epidemiological research, the validity of the diagnosis is essential to draw valid conclusions from studies using registry data. In Denmark, two nationwide data sources are available with regard to RA patients: the nationwide DANBIO clinical register for inflammatory arthritides, including rheumatoid arthritis (RA), is a dedicated clinical quality and research registry used in routine care by all departments of rheumatology in Denmark. ${ }^{4}$ The Danish National Patient Registry (DNPR) is a nationwide, administrative registry that covers all hospitalizations and outpatient visits. $^{5}$ In DANBIO, detailed longitudinal clinical information about representative disease course, patient-reported outcomes (PRO), joint examinations, and medical 
treatment are collected as part of routine care, whereas DNPR data are collected for administrative purposes.

In an earlier study, the validity of RA diagnoses in DNPR during the period 1977-2001 was investigated by comparing DNPR data with the diagnoses registered in patient records. The results showed that the proportion of valid RA diagnoses in DNPR varied between 20\% and 90\%. Correct diagnoses were more often found in patients with frequent contacts and in patients seen at departments of rheumatology. ${ }^{6}$ The DANBIO registry was established in October 2000 and, therefore, had no data available for comparison. ${ }^{4}$ The annual DANBIO report estimates the completeness of patients with the RA diagnoses in DANBIO compared to DNPR at $\sim 85 \%$ for the recent years ${ }^{7}$; however, no concurrent validation of the diagnoses in both registries has previously been undertaken.

Accurate knowledge about the overlap and validity of RA diagnoses is important for future research based on these two registries. The objective of this study was to study the validity of the RA diagnosis (positive predictive value [PPV]) and to estimate the completeness of relevant RA cases in each registry.

\section{Materials and methods The DANBIO registry and DNPR}

The DANBIO registry (full name: DANBIO - The Danish Rheumatologic Database) contains clinical and treatment data, which are entered by rheumatologists as part of routine care during the disease course of patients with inflammatory rheumatic diseases. Patient-reported outcomes (PROs) entered by patients from touchscreens in the waiting area in the outpatient clinics - are also collected. DANBIO data are used for the monitoring of treatment quality and for research purposes. ${ }^{8-12}$ Initially, DANBIO was a voluntary registry only including patients treated with biological drugs; but, since 2006, it has been mandatory to also include newly referred RA patients regardless of treatment and disease duration. ${ }^{4}$ By spring 2015, more than 26,000 patients registered with RA had been included in DANBIO. ${ }^{7}$

In Denmark, hospital-based healthcare is funded through public taxation. Since 1977, all hospitals are required to report administrative data, including diagnostic information, for inpatients to the DNPR and, from 1995, also for outpatients..$^{5}$ During the study period, all diagnoses were coded using the ICD-10 classification system. ${ }^{13}$

Application of unique personal identifiers (assigned to all Danish citizens at birth or when achieving citizenship) enables accurate linkage of individuals between registries.

\section{Study population}

In January 2013, data were obtained from both the DANBIO and the DNPR, for all patients registered with an RA diagnosis during the period between 2001 and 2011. We included the following ICD-10 diagnostic codes: M05.9 (seropositive RA), M06.0 (seronegative RA), M06.8 (other types of RA), and M06.9 (unspecified RA).

Based on the data extracted from the two registries, we identified incident RA patients with their first RA diagnosis in 2011 (by excluding all patients with a registered RA diagnosis during 2001-2010). A total of 2,298 unique patients were identified. From this population, we grouped patients into three categories: overlapping if they were registered in DANBIO and DNPR, DANBIO only if registered in DANBIO only, and DNPR only if registered in DNPR only (Figure 1).

In order to increase the chances of obtaining true RA patients, the DNPR population was limited to patients who had a visit with a RA diagnosis at a hospital department of rheumatology in 2011, and who had at least one additional visit with a RA diagnosis within 90 days after the first visit. This was based on the methods used in the former validation study, which showed that the chances of retrieving true RA cases was much higher when requiring two RA registrations in DNPR rather than only one, and with only a minor additional gain in restricting to three registrations. ${ }^{6}$ The filter reduced our population to 1,678 unique patients in total (Figure 1).

\section{Review of patient records}

For the identified population of patients with registered RA diagnoses, one rheumatologist at each hospital department retrieved the medical records. Based on the information available in the patient records around the time of diagnosis, the rheumatologists categorized patients as having RA based on the rheumatologists' expert opinion. Before data collection, two rheumatologic specialists at Gentofte University Hospital evaluated the ACR-87 criteria $^{14}$ and the expert opinion method for categorizing patients as having RA for 80 patients and found a perfect match.

For patients who could not be confirmed as having RA, a relevant other diagnosis or a possible explanation for lack of registration in either DANBIO or DNPR was recorded. Rheumatologists were blinded for the registry details of the patients. In total, 1,532 patient records (Figure 1) at 21 hospital departments were reviewed. Of the remaining 146 patient records, 57 could not be located at the responsible hospital, and the remaining 89 patients were treated at 


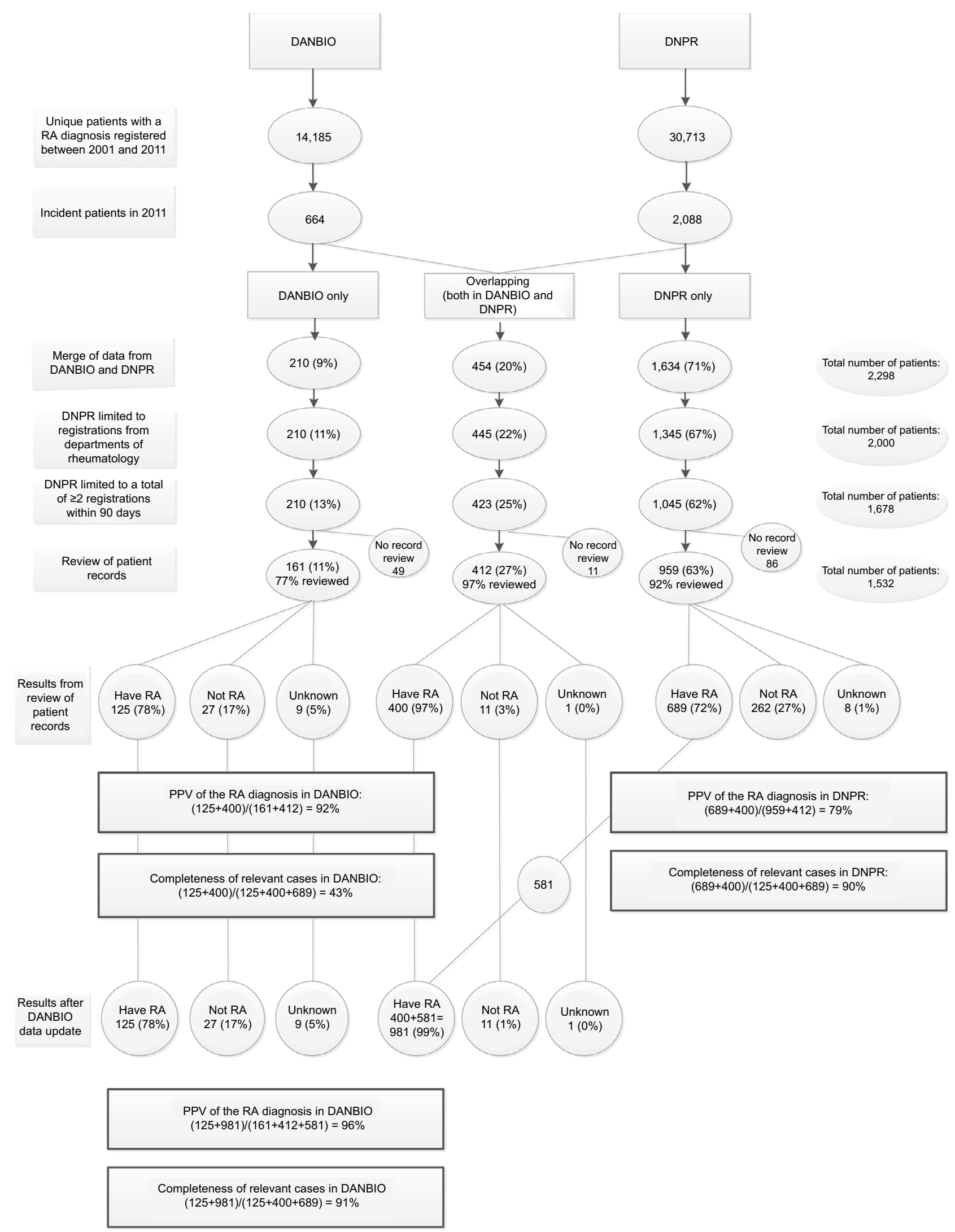

Figure I Results from data retrieval and merged data between the Nationwide Clinical Register for Patients with Rheumatoid Arthritis (DANBIO) and the Danish National Patient Registry (DNPR), as well as from medical file review; $n(\%)$, positive predictive values (PPV), and registry completeness of relevant RA cases. 
three hospitals that did not participate in the study (data not shown).

\section{Update of data}

In 689 RA patients registered in 2011, the review of patient records confirmed a valid RA diagnosis in DNPR, but the patients were not in the initial DANBIO dataset (Figure 1). As the focus on registration into DANBIO has increased over time, we retrieved updated data from DANBIO primo 2015 to investigate whether these patients had been registered in DANBIO after the initial data extraction.

\section{Analysis}

Based on the review of the patient records, the PPV of the RA diagnoses in DANBIO and DNPR, respectively, were calculated as the number of patients with a verified RA diagnosis in each registry divided by all patients with records reviewed from DANBIO and DNPR, respectively. The completeness of relevant RA cases was calculated for each registry as the number of verified RA patients in DANBIO and DNPR, respectively, compared to the total number of verified patients from both registries (Figure 1). This should be interpreted as an estimation of the registry completeness of true RA patients treated in hospital settings, as the two registries do not hold data of RA cases treated only in primary care.

By using the chi-square test and OLS regression with age in years as continuous variable and data source as dichotomous variable, we tested whether a difference in the distribution of gender or age existed between the three groups of patients registered in DANBIO only, DNPR only, and overlapping based on data before record review.

\section{Ethics}

According to the rules at the time, the Danish Data Protection Agency was notified of the project's use of data.

\section{Results}

Results from the data retrieval and review of patient records are shown in Figure 1. In 2011, a total of 664 incident patients with a diagnosis of RA were identified in DANBIO, and 2,088 incident patients were identified in the DNPR. After merging data from the two data sources, and restricting patients from DNPR to include only those treated at hospital departments of rheumatology and those with two or more outpatient visits with RA as diagnosis, the number of patients in DANBIO only was 210 , overlapping patients (both in DANBIO and in DNPR) was 423, and patients in DNPR only was 1,045 . No differences were found in the distribution of gender or age between the three groups of patients (data not shown). The rheumatologists conducted reviews of the patient records in $77 \%, 97 \%$, and $92 \%$ of patients in the three groups (DANBIO only, overlapping patients, and DNPR only; Figure 1).

The PPV of the RA diagnosis (as defined in Figure 1) was $92 \%$ for the DANBIO population and $79 \%$ for the DNPR population. With the inclusion of the updated DANBIO dataset, the PPV of the RA diagnosis in DANBIO was 96\% (581 patients with verified RA from DNPR only were also identified in DANBIO with this update; Figure 1). The registry completeness of relevant RA cases for DANBIO was $43 \%$ based on the initial data, and $91 \%$ based on the updated data. For DNPR, it was 90\% (Figure 1).

Table 1 shows the diagnoses in patients who, by review, were found to have a non-RA diagnosis. Osteoarthritis,

Table I Distribution of diagnoses from patient records of non-RA patients in the Nationwide Clinical Register for Patients with Rheumatoid Arthritis (DANBIO) and the Danish National Patient Registry (DNPR), (n)

\begin{tabular}{llll}
\hline Diagnosis & DANBIO only & Both in DANBIO and DNPR & DNPR only \\
\hline Arthralgia & 2 & 5 & 36 \\
Crystal arthropathy & 0 & 0 & 16 \\
Psoriatic arthritis & 0 & 1 & 34 \\
Osteoarthritis/spondyloarthritis & 1 & 3 & 39 \\
Spondyloarthropathy & 0 & 0 & 11 \\
Juvenile arthritis & 0 & 0 & 7 \\
Polymyalgia & 1 & 0 & 16 \\
Reactive arthritis & 0 & 0 & 13 \\
Oligo/polyarthritis & 5 & 1 & 20 \\
Soft tissue rheumatism & 0 & 0 & 5 \\
Connective tissue diseases & 1 & 0 & 10 \\
Palindromic arthritis & 0 & 0 & 7 \\
Other & 3 & 0 & 23 \\
Missing & 14 & 1 & 25 \\
Total & 27 & 11 & 262 \\
\hline
\end{tabular}


psoriatic arthritis, and arthralgia were the most frequent non-RA diagnoses.

\section{Discussion}

This study showed that $96 \%$ of RA patients registered in DANBIO and $79 \%$ of those in the DNPR were valid cases in the calendar year 2011. The completeness of relevant RA cases in each registry was estimated to be $\sim 90 \%$ when a lag time between the period of interest and time of retrieving data was added.

The high validity of the diagnosis in DANBIO and the high percentage of false positive (one in five cases) in DNPR, even after applying rather strict filters, are important for future epidemiologic research projects in Danish RA patients. DANBIO is a clinical tool that gives an overview of patients' disease course and is used by all rheumatologists providing routine care. The rheumatologists are also responsible for recoding data, if diagnoses are revised, and this contributes to the very high proportion of valid cases in DANBIO.

The discrepancy in numbers of incident RA cases between DANBIO and DNPR in the 2011 dataset was partly explained by a high false-positive rate of RA diagnoses in DNPR. Moreover, there was a delay in the registration of RA diagnoses in DANBIO as compared to the DNPR, probably because it often takes the clinician several months to establish the correct diagnosis, and the patient is only registered with the specific diagnosis RA in DANBIO thereafter. In contrast, the DNPR is an administrative registry, and the RA diagnosis is sometimes registered before it has been clinically confirmed - that is, at the time of referral. Some discrepancies between dates of diagnosis were found. In DANBIO, the month and year of diagnosis is entered by rheumatologists as a core variable. In the DNPR, the diagnosis is registered on the date of first in-or out-patient contact; therefore, the date of first contact may provide a poor indicator of the true diagnosis date.

The PPV of the RA diagnosis in DNPR was 79\% when a previously developed filter was used for retrieving DNPR cases. ${ }^{6}$ For comparison, a recent study estimated the PPV of the Systemic Lupus Erythematosus diagnosis in DNPR to be $\sim 70 \% .{ }^{15}$ Other recent validation studies of DNPR reported that the PPV was $80 \%-82 \%$ for acute coronary syndrome, ${ }^{16}$ $81 \%$ for epilepsy, ${ }^{17}$ and $92 \%$ for chronic obstructive pulmonary disease $92 \%,{ }^{18}$ based on specialists' review of patient records. The Swedish National Patient Register reported a PPV of the RA diagnosis of $91 \%$, using an algorithm that also included prescription of disease modifying drugs from the Swedish Prescribed Drug Register. ${ }^{19}$ A Canadian study found a PPV for RA of $51-83 \%$ in administrative data from both primary and secondary healthcare setups. ${ }^{20}$

We evaluated the RA diagnosis through review of a large number of patient records from nationwide data sources. It is, however, a limitation that milder cases of RA treated in the primary care sector by general practitioners are not included in the registries, but a strength that the practicing rheumatologists use and increasingly report into DANBIO. ${ }^{7}$ In DANBIO's annual quality report, the number of newly registered patients in DANBIO (rather than newly diagnosed) has been compared to the DNPR with no validation of the RA diagnosis. The proportions of RA patients in DNPR registered in DANBIO were calculated to be $79 \%, 83 \%$, and $85 \%$, respectively, for the period $2011-2013,{ }^{7}$ which indicates that registration practice, in general, has improved. This is in accordance with an increasing number of RA patients registered in DANBIO ( $>26,000$ registered patients by spring $2015^{7}$ ), as a result of an increased focus on correct registrations in both DANBIO and DNPR. The prevalence of RA in Denmark has previously been estimated to be 35,000 based on self-report surveys, ${ }^{21}$ which supports that a minor part of patients with RA are not followed in hospitals. ${ }^{22}$ It should, however, be noted that data on incident RA cases from DANBIO before 2006 will probably have lower completeness of cases than reported in this study, because DANBIO registration of newly referred RA patients and RA patients receiving non-biological treatment first became mandatory in 2006.

It is an important finding for future epidemiologic research projects in Danish RA patients that the DANBIO registry provided a high proportion of valid diagnosis, although with a delay in registration of incident cases.

\section{Conclusion}

Based on a review of 1,532 incident cases, our study of two nationwide registries showed a high PPV of the RA diagnosis in DANBIO (96\%) and a moderate PPV in DNPR (79\%). The registry completeness of patients treated in hospital care was estimated to be $\sim 90 \%$ in both registries.

\section{Acknowledgments}

The Hospital Departments of Rheumatology in Demark and the private rheumatologic clinics are acknowledged for their contribution to DANBIO. Zitelab ApS is acknowledged for the technical development and support of the DANBIO register, and the Danish Regions for financial support.

\section{Disclosure}

The authors report no conflicts of interest in this work. 


\section{References}

1. Lee DM, Weinblatt ME. Rheumatoid arthritis. Lancet. 2001; 358(9285):903-911.

2. Løppenthin K, Esbensen BA, Østergaard M, Ibsen R, Kjellberg J, Jennum P. Welfare costs in patients with rheumatoid arthritis and their partners compared with matched controls: a register-based study. Clin Rheumatol. 2017;36(3):517-525.

3. Hetland ML, Jensen DV, Krogh NS. Monitoring patients with rheumatoid arthritis in routine care: experiences from a treat-to-target strategy using the DANBIO registry. Clin Exp Rheumatol. 2014;32(5 Suppl 85):S141-146.

4. Ibfelt EH, Jensen DV, Hetland ML. The Danish nationwide clinical register for patients with rheumatoid arthritis: DANBIO. Clin Epidemiol. 2016;8:737-742. eCollection 2016.

5. Schmidt M, Schmidt SA, Sandegaard JL, Ehrenstein V, Pedersen L, Sørensen HT. The Danish National Patient Registry: a review of content, data quality, and research potential. Clin Epidemiol. 2015;7:449-490.

6. Pedersen M, Klarlund M, Jacobsen S, Svendsen AJ, Frisch M. Validity of rheumatoid arthritis diagnoses in the Danish National Patient Registry. Eur J Epidemiol. 2004;19(12):1097-1103.

7. DANBIO's National Clinical Quality report 2014 (report in Danish, Available from: www.danbio-online.dk). 2015. Copenhagen. Accessed September 29, 2017.

8. Dreyer L, Mellemkjær L, Andersen AR, et al. Incidences of overall and site specific cancers in TNF $\alpha$ inhibitor treated patients with rheumatoid arthritis and other arthritides - a follow-up study from the DANBIO Registry. Ann Rheum Dis. 2013;72(1):79-82.

9. Gabay C, Riek M, Hetland ML, et al. Effectiveness of tocilizumab with and without synthetic disease-modifying antirheumatic drugs in rheumatoid arthritis: results from a European collaborative study. Ann Rheum Dis. 2016;75(7):1336-1342.

10. Glintborg B, Sørensen IJ, Loft AG, et al; all departments of rheumatology in Denmark. A nationwide non-medical switch from originator infliximab to biosimilar CT-P13 in 802 patients with inflammatory arthritis: 1 -year clinical outcomes from the DANBIO registry. Ann Rheum Dis. 2017;76(8):1426-1431.

11. Hansen SM, Hetland ML, Pedersen J, Østergaard M, Rubak TS, Bjorner JB. Work ability in rheumatoid arthritis patients: a register study on the prospective risk of exclusion and probability of returning to work. Rheumatology (Oxford). 2017;56(7):1135-1143.
12. Hetland ML, Christensen IJ, Tarp U, et al; All Departments of Rheumatology in Denmark. Direct comparison of treatment responses, remission rates, and drug adherence in patients with rheumatoid arthritis treated with adalimumab, etanercept, or infliximab: results from eight years of surveillance of clinical practice in the nationwide Danish DANBIO registry. Arthritis Rheum. 2010;62(1):22-32.

13. World Health Organization. International Statistical Classification of Diseases and Related Health Problems 10th Revision. 2016. Available from: http://www.who.int/classifications/icd/en/. Accessed September $29,2017$.

14. Arnett FC, Edworthy SM, Bloch DA, et al. The American Rheumatism Association 1987 revised criteria for the classification of rheumatoid arthritis. Arthritis Rheum. 1988;31(3):315-324.

15. Hermansen ML, Lindhardsen J, Torp-Pedersen C, Faurschou M, Jacobsen S. Incidence of systemic lupus erythematosus and lupus nephritis in Denmark: a nationwide cohort study. J Rheumatol. 2016;43(7):1335-1339.

16. Joensen AM, Jensen MK, Overvad K, et al. Predictive values of acute coronary syndrome discharge diagnoses differed in the Danish National Patient Registry. J Clin Epidemiol. 2009;62(2):188-194.

17. Christensen J, Vestergaard M, Olsen J, Sidenius P. Validation of epilepsy diagnoses in the Danish National Hospital Register. Epilepsy Res. 2007;75(2-3):162-170.

18. Thomsen RW, Lange P, Hellquist B, et al. Validity and underrecording of diagnosis of COPD in the Danish National Patient Registry. Respir Med. 2011;105(7):1063-1068.

19. Waldenlind K, Eriksson JK, Grewin B, Askling J. Validation of the rheumatoid arthritis diagnosis in the Swedish National Patient Register: a cohort study from Stockholm County. BMC Musculoskelet Disord. 2014;15:432.

20. Widdifield J, Bombardier C, Bernatsky S, et al. An administrative data validation study of the accuracy of algorithms for identifying rheumatoid arthritis: the influence of the reference standard on algorithm performance. BMC Musculoskelet Disord. 2014;15:216.

21. Sundhedsstyrelsen. Leddegigt - medicinsk teknologivurdering af diagnostik og behandling (report in Danish). 2002. Available from: https:// www.sst.dk/da/udgivelser/2002/leddegigt. Accessed September 29, 2017.

22. Pedersen JK, Kjaer NK, Svendsen AJ, Hørslev-Petersen K. Incidence of rheumatoid arthritis from 1995 to 2001: impact of ascertainment from multiple sources. Rheumatol Int. 2009;29(4):411-415.
Clinical Epidemiology

\section{Publish your work in this journal}

Clinical Epidemiology is an international, peer-reviewed, open access, online journal focusing on disease and drug epidemiology, identification of risk factors and screening procedures to develop optimal preventative initiatives and programs. Specific topics include: diagnosis, prognosis, treatment, screening, prevention, risk factor modification,

\section{Dovepress}

systematic reviews, risk and safety of medical interventions, epidemiology and biostatistical methods, and evaluation of guidelines, translational medicine, health policies and economic evaluations. The manuscript management system is completely online and includes a very quick and fair peer-review system, which is all easy to use. 